\title{
A new dynamic-XPS end-station for beamline P04 at PETRA III/DESY
}

\author{
Sergey V. Babenkov ${ }^{a}, *$, Victor Y. Aristov ${ }^{\mathrm{b}, \mathrm{c}}$, Olga V. Molodtsova ${ }^{\mathrm{a}}$, Leif \\ Glaser $^{\mathrm{a}}$, Ivan Shevchuk ${ }^{\mathrm{a}}$, Frank Scholz ${ }^{\mathrm{a}}$, Jörn Seltmann ${ }^{\mathrm{a}}$, and Jens Viefhaus ${ }^{\mathrm{a}}$ \\ ${ }^{a}$ Deutsches Elektronen-Synchrotron DESY, Notkestraße 85, 22607 Hamburg, Germany \\ ${ }^{b}$ Institute of Solid State Physics of Russian Academy of Sciences, Chernogolovka 142432, \\ Russia \\ ${ }^{c}$ Institut für Theoretische Physik, Universität Hamburg, Jungiusstraße 9, D-20355 \\ Hamburg, Germany
}

\begin{abstract}
We report on a new dynamic-XPS end-station for real-time investigations of advanced materials. The end-station is based on a new Argus hemispherical electron spectrometer with high speed detection system. In combination with the high brilliance XUV beamline P04 at PETRA III it provides users at PETRA III a unique tool for fast (down to $0.1 \mathrm{sec} /$ spectrum) and detailed investigations compared to existing XPS devices at other beamlines.
\end{abstract}

Keywords: Electron spectrometer; Dynamic-XPS; Snapshot; High resolution.

\section{Introduction}

Photoelectron spectroscopy is a well established and commonly used technique to obtain information about electronic structure of surface of solid state materials and rare gases [1]. Technical progress in construction of X-ray sources 5 as well as in manufacture of electron spectrometers and detectors provides researchers a possibilities to investigate more complex and advanced materials. For instance, growth processes of graphene on several substrates [2-5], metalorganic interfaces formation [6, 7], fabrication of hybrid organic-inorganic systems $[8,9]$ and etc. From the other hand, the synthesis of such materials may

\footnotetext{
* Principal corresponding author

Email address: sergey.babenkov@desy.de (Sergey V. Babenkov)
} 
occasionally involve fast processes, e.g. reaction kinetics [10-12], which consequently should be precisely controlled and characterised. The problem could be solved by measuring dynamic photoelectron spectra with acquisition times per spectrum in the millisecond time resolution [11].

One way to realise experiments with sufficient time resolution is the combination of a high brightness beamline with a new hemispherical analyser Argus (Omicron NanoTechnology GMBH, Germany). This analyser uses a 128 channel multi-anode detector, which can generate photoelectron spectra in both traditional scanning as well as a so-called snapshot mode (128 channels of the electron detector covered the whole core level peak). The snapshot mode allows dynamic data acquisition in the millisecond regime. The analyser energy resolution (30 meV at $250 \mathrm{eV}$ kinetic energy) is in line with the beamline specifications and allows sufficient resolution for any high resolution time dependent XPS experiments. The described end-station is designed, built up and installed into the beamline P04 (PETRA III, DESY, Hamburg). Initial experiments success-

25 fully demonstrated the potential of obtaining dynamic photoelectron spectra at P04 for in-situ growth studies, which could be applied to other research fields $[13,14]$.

\section{Design of experimental station}

\subsection{Overview of instrument}

30

The experimental station consists of 3 main parts (Fig. 1). The middle one is a small ultra-high vacuum (UHV) analysis chamber (base pressure below 1 $\mathrm{x} 10^{-10}$ mbar) equipped with the hemispherical electron-analyser, which is connected on the right side of the analysis chamber (Fig. 1). The analysis chamber includes a manipulator with 4 degrees of freedom $(x, y, z$ directions and polar 35 angle around the $z$ axis). The main part of manipulator is a special designed head, whose design is described below (see sec.2.3). The analysis chamber is also equipped with a quartz microbalance, an effusive capillary gas source and an evaporator of metals. The metal evaporator and an effusive capillary gas 
source are placed within small angle with respect to photon beam $\left(12.5^{\circ}\right)$. This arrangement allows to perform in-situ deposition of metal and apply gas atmosphere in the vicinity of sample surface, with only barely increases the base pressure during measurements. Furthermore, CF-40 flanges allow to mount additional devices.

To the left side of Figure 1 UHV preparation chamber (base pressure below ${ }_{45} 1 \times 10^{-10} \mathrm{mbar}$ ) is attached to the analysis chamber. This chamber is equipped with a quartz microbalance, a sputtering gun, an organic evaporator and a linear manipulator with propose-built sample transfer system. In addition, there is a sample garage for a number of holders and a gas inlet system as well as a fast entry lock window which provides the possibility of fast introduction of new

50 samples, if required. Design of sample holder (for details see sec.2.3) provides possibility to control sample temperatures in the range from 300 up to $1700 \mathrm{~K}$ during preparation procedure.

\subsection{The hemispherical electron analyser.}

Dynamic XPS experiments aim for monitoring of time depended processes 55 e.g. the temperature stimulated Graphene growth on the surface of cubic$\mathrm{SiC} / \mathrm{Si}$. Specifically, in order to observe irreversible processes with sufficient time resolution and data quality high photon flux and multi bunch mode from the synchrotron source, optimized spectrometer transmission and an adequate

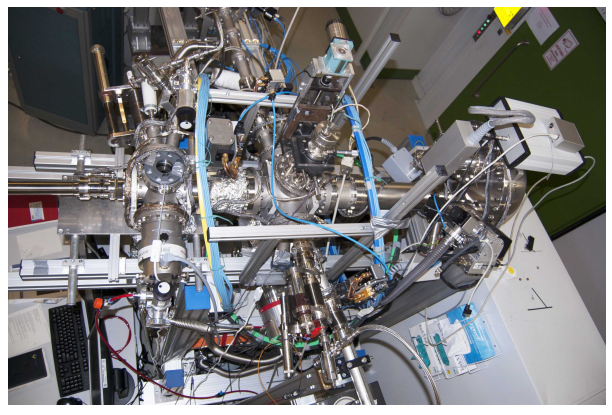

Figure 1: Experimental setup: (from left to right) sample preparation chamber, analysis chamber, spectrometer Argus. 


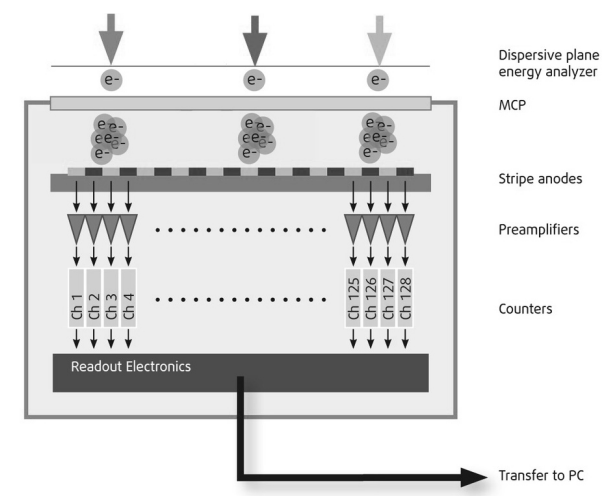

Figure 2: Detector scheme: each electron impact starts a cascade of electrons propagating through the MCP stack. The amplified electron signals are collected by a stripe anode array which is connected to 128 dedicated amplifiers and counters are situated in UHV closely connected to the stripe anode for optimum signal to noise ratio.

detection system are essential for such experiment.

Other time resolved experiments [15] demand for sophisitcated experimental setups which allow ultra fast time resolution into the fsec regime. Another commonly used approach is a pump-probe setup which utilizes synchronized data acquisition with the pump and probe light pulses. A key advantage of such experiments is the repeatability of the process which allows accumulating signals over multiple repeats and as result moderate duty cycles can be accepted $[16]$.

In the presented dynamic XPS end station design a modern high transmission turnkey hemispherical electron spectrometer system (Argus) with small spot lens for XPS, imaging XPS and AES applications has been implemented. It is based on an electrostatic hemispherical energy analyser with a mean radius of $124 \mathrm{~mm}$. The analyser focuses electrons in both angular directions, from the entrance onto the exit plane.

Key to the experimental setup is a new type of multianode detector [17] which uses a microchannel plate (MCP) to amplify the electron signals (Fig. 2).

75 However in contrast to other multi channel detectors [18] the amplified signals impact on an on-chip stripe-anode array which is hardwired to 128 independent 
working pulse counters all implemented in UHV allowing for massive parallel detection. Compared to other detection concepts the proximity of anode and pulse counting electronics in UHV is highly optimized. As a result the sensitivity and the signal to noise performance of the detector are excellent.

The detector has demonstrated quantitative XPS results with peak count rates larger than 100 Mcps [17]. Special care has been taken to calibrate the detector in order to allow quantitative snapshot XPS performance. The detector has been tested in snapshot mode signature free up to the highest count rates.

For time resolved experiments the detector allows continuous run in snapshot mode with dwell times in the msec time regime. The analyser is operated by MATRIX Automated Task Environment (MATE) software (for details see $\operatorname{Ref}[23])$.

\subsection{Manipulator head of sample holders and sample transfer system}

The design of sample holder, manipulator head and sample transfer system is based on ASPHERE III Setup sample holder system (University of Kiel) $[19,20]$. The stainless steel manipulator head has 3 slots for sample holders and is connected directly to manipulator rod (Figure 3). The rotation axis of manipulator coincides with sample surface, thereby it allows it to rotate a

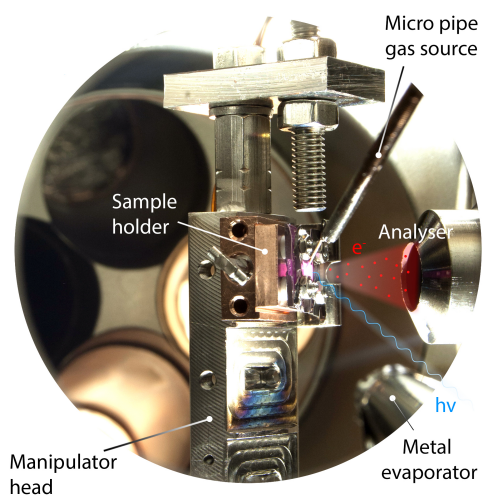

Figure 3: Current view of analysis chamber. The sample holder with heated piece of $\mathrm{SiC} / \mathrm{Si}$ wafer (around $800^{\circ} \mathrm{C}$ ), the gas source in front of sample surface, as well as the electron analyser entracne aperture and metal evaporator can be seen. 
Table 1: Parameters of Soft X-Ray beamlines with end stations based on delay-line detection system.

\begin{tabular}{llllll}
\hline Beamline & $\begin{array}{l}\text { Photon energy } \\
\text { range eV }\end{array}$ & Resolving power & $\begin{array}{l}\text { Photon flux } \\
\text { photons/s@RP }\end{array}$ & $\begin{array}{l}\text { Spot size } \\
\mu \mathrm{m} \times \mu \mathrm{m}\end{array}$ & Polarization \\
\hline XUV P04, DESY & $\begin{array}{l}250-3000 \\
50-3000\end{array}$ & $3 \times 10^{4} @ 300 \mathrm{eV}$ & $5 \times 10^{12} @ 1 \times 10^{4}$ & $\begin{array}{l}\text { Vertical } 10 \mu \mathrm{m} \\
\text { Horizontal } 10 \mu \mathrm{m}\end{array}$ & $\begin{array}{l}\text { Circular } \\
\text { Linear }\end{array}$ \\
$\begin{array}{l}\text { Expected in 2015 } \\
\text { SuperESCA, Elettra }\end{array}$ & $90-1800$ & $3 \times 10^{4} @ 400 \mathrm{eV}$ & $\begin{array}{l}1 \times 10^{11} @ 1 \times 10^{4} \\
4 \times 10^{12} @ 5 \times 10^{3}\end{array}$ & $\begin{array}{l}10 \times 100 \\
10 \times 40\end{array}$ & $\begin{array}{l}\text { Circular, linear } \\
\text { Circular, linear }\end{array}$ \\
\hline
\end{tabular}

sample without lateral shift. The upper slot is equipped with direct current heating and $\mathrm{LN}_{2}$ cooling stages that allow to control the sample temperature in the range from 100 up to $1700 \mathrm{~K}$. The contact sheets are arranged in a way that allows spectra acquisition in a wide range of the electron emission angles $\left(0-77.5^{\circ}\right)$ while temperature changing. At the same time, 2 additional slots provide possibility to investigate samples at room temperature.

Figure 3 represents the current view of manipulator head and sample holder with heated piece of $\mathrm{SiC} / \mathrm{Si}$ wafer. In addition, the position of capillary gas source, analyser and metal evaporator is indicated.

\subsection{Key parameters of the setup}

The experimental station is installed into Variable Polarization XUV beamline P04 at PETRA III. Table 1 presents of most important parameters of the beamline P04 and others, which are also using delay-line detection system $[11,21,22]$. Photon energy range of beamline P04 is $250-3000 \mathrm{eV}$. Optical mode, which makes use of the off axis radiation of the undulator, gives a potential to expand this range down to $50 \mathrm{eV}$. Resolving power (RP) is more than $10^{4}\left(>3 \times 10^{4} @ 300 \mathrm{eV}\right)$. Photon flux is $5 \times 10^{12}$ photons/s at resolving power $10^{4}$. Variable polarisation in $2013 / 14$ is only between left- and right-handle circularly polarized. Spot size at sample is down to $10 \mu \mathrm{m}$ (2013/14 in the vertical only). This combination of parameters makes the beamline most suitable for dynamic-XPS experiments, in comparison with other soft X-Ray beamlines. 


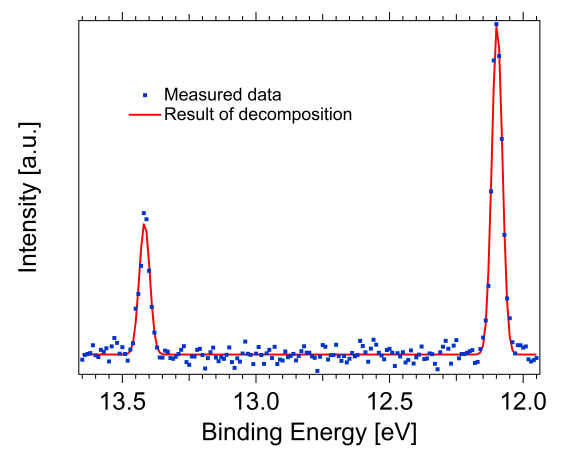

Figure 4: Experimental spectrum of Xe $5 p$ measured at $265 \mathrm{eV}$ photon energy. Blue dots are experimental data. Red line is decomposition result of experimental data.

The energy resolution of the spectrometer was confirmed to be smaller than $30 \mathrm{meV}$ for kinetic energies of about $250 \mathrm{eV}$ using a Xenon gas phase target and the Xe $5 p$ electron lines (Fig. 4). Similar value $(25 \mathrm{meV})$ was estimated from $\mathrm{Au} 4 f$ core level deconvolution.

The experimental spectra were recorded in snapshot mode (See sec. 3). Apart from scanning and snapshot modes, the design of spectrometer provides a possibility to scan a sample surface in XPS range without mechanical shift of the sample ("dynamic mode" of XPS). This mode allows to understand complex local chemistry and to obtain local PE spectra along the surface of analysed sample with lateral resolution better than $60 \mu \mathrm{m}[23,24]$.

\section{Time resolved in-situ observation of temperature stimulated graphene growth onto the surface of cubic-SiC/Si}

Following our recent work $[5,25-27]$ the key attention was paid to investigation of graphene. The temperature stimulated self-organised growth of carbon atoms into graphene structure is well studied on different substrates and by different methods [5, 25-32]. The methods are very laborious and consist of several steps (sublimation of $\mathrm{Si}$, surface reconstruction of carbon, hybridisation of carbon-carbon bonds, etc) which should be successively tracked during preparation procedure [25]. There are different parameters (heating time of sample, 


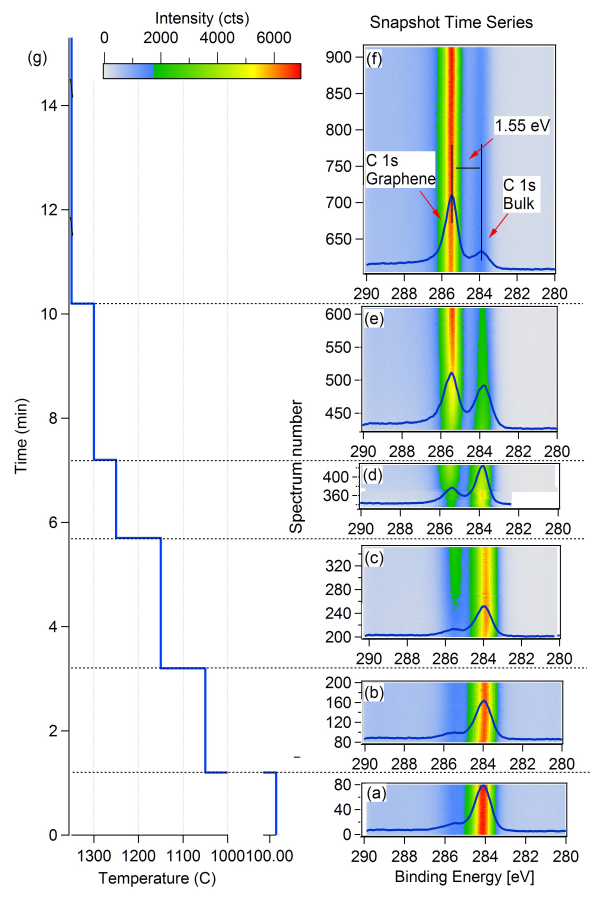

Figure 5: (a-f) Time evolution of $\mathrm{C} 1 s$ core level snapshot PE spectra recorded during heating process. More than 900 snapshot spectra have been recorded with acquisition time of 1 sec/spectrum. A one of single snapshot spectrum at critical stages is shown. (g) Temperature of sample during the measurements.

applying surrounding atmosphere, heating temperature, etc) which could be changed during preparation procedure $[25,33]$. There are no experiments with direct observation of real time preparation graphene layers and no methods to control an amount of graphene layers during preparation procedure.

Taken this into account, we followed for temperature stimulated process of in-situ graphene synthesis on piece of cubic-SiC/Si wafer by measuring of $\mathrm{C}$ $1 s$ photoemission spectra in snapshot mode. The sample was annealed at 600$700^{\circ} \mathrm{C}$ during 1 hour to outgas the surface. Also the sample was flashed at $1100^{\circ} \mathrm{C}$ to remove silicon oxide from the surface. During the measurements we applied a direct current to sample to heat it up to $1350^{\circ} \mathrm{C}$ (Fig. 5(g)). 145 More than 900 spectra of C $1 s$ core level with acquisition time $1 \mathrm{sec} /$ spectrum 
were recorded during 15 minutes, using $750 \mathrm{eV}$ photon energy and $70 \mathrm{eV}$ pass energy (Fig. 5(a)-(e)). A one of single snapshot spectrum at critical stages is shown. Despite of quite high sample temperature and photon energy, as well as short time of spectrum acquisition, the spectra are well pronounced and allow to observe the shape and ratio of peak during the preparation procedure. Due to current applied to the sample, the acquired spectra were shifted. In order to compensate this, the $\mathrm{C} 1 s \mathrm{SiC}$ peak was used as a reference.

At the first stage (Fig. 5(a)) of preparation procedure one can see a strong peak which corresponds to carbon in SiC bulk. Increasing the temperature (Fig. $5(\mathrm{~b})-(\mathrm{e}))$ gives rise to a small additional component of the $\mathrm{C} 1 s$ line shifted by $1.55 \mathrm{eV}$ to higher binding energy. At the same time the bulk component is decreasing. This corresponds to a process of sublimation of $\mathrm{Si}$ atoms and a graphitisation of the surface [25]. Finally, at the last stage of preparation procedure (Fig. 5(f)), the temperature of sample has reached $1350^{\circ} \mathrm{C}$. At this temperature the carbon-carbon bonds undergo a reconstruction to $\mathrm{sp}^{2}$ hybridisation which correspond to graphene formation [25]. After this procedure we ex-situ have moved our sample to another experimental station for LEED measurements, which proved the existence of a synthesised graphene layers on the $\mathrm{SiC} / \mathrm{Si}$ wafer $[25,32]$.

\section{Summary}

The new dynamic-XPS end station is installed into beamline P04 (PETRA III, DESY), commissioned and available for regular users. The combination of beam line and end station allows to measure XPS spectra as a function of varying conditions, using both scanning and snapshot modes, with possibility to parallel snapshot measurements of several core levels. Consequently, it opens access to real time investigations of fast flow processes and quantitative analysis of surface layers. For instance, one can follow the reaction kinetics by observation of spectrum shape or control of the deposition rate and amount of a given substance by monitoring the intensity or ratio of peaks in the snapshot spectra. Since 
the beamline has possibility to smoothly change a photon energy, a NEXAFS technique is available. Moreover, the beamline has several polarisation mode and consequently there is a possibility to measure XMCD.

The shown results proof effectivity of the dynamic-XPS end station and the ability to identify critical phases of fast real-time processes by following the evolution of photoemission core level spectra.

To our knowledge there is no alternative beamline - end station combination in terms of spectrum acquisition speed, photon flux, photon energy range and photon energy resolution. The beamline parameters (linear polarisation mode, beam focusing in horizontal plane, extension of photon energy range down to $50 \mathrm{eV}$ ) and new possibilities of end station (interconnection with beamline and speed of the detection system) will be available very soon.

\section{Acknowledgments}

We want to thank Omicron NanoTechnology GMBH for providing the Argus analyser, especially Konrad Winkler and Georg Prümper for support, as well as Wolfgang Drube for the loan of equipment. We acknowledge support by the P04 team and also all DESY-FS service groups and workshops. Moreover, we thank the ASPHERE III collaboration, especially Arndt Quer, Erik Kröger, Matthias Kalläne, Lutz Kipp (University of Kiel) as well as Hendrik Bentmann and Cristoph Seibel (University of Würzburg) for valuable advice concerning the design of the experimental stations and discussion of the results.

This work was supported by RFBR grants №13-02-00818, №14-02-00949 and the BMBF-Project №05K12GU2, PSP-Element №U4606BMB1211.

\section{References}

[1] J. F. Watts, J. Wolstenholme, An Introduction to Surface Analysis by XPS and AES, 2014. 
[2] K. S. Kim, Y. Zhao, H. Jang, S. Y. Lee, J. M. Kim, K. S. Kim, J.-H. Ahn, P. Kim, J.-Y. Choi, B. H. Hong, Nature 457 (2009) 706. doi:10.1038/ nature07719.

[3] A. Reina, X. Jia, J. Ho, D. Nezich, H. Son, V. Bulovic, M. S. Dresselhaus, J. Kong, Nano Letters 9 (2009) 30. doi:10.1021/n1801827v.

[4] C. Berger, Z. Song, X. Li, X. Wu, N. Brown, C. Naud, D. Mayou, T. Li, J. Hass, A. N. Marchenkov, E. H. Conrad, P. N. First, W. A. de Heer, Science 312 (2006) 1191. doi:10.1126/science.1125925.

[5] V. Y. Aristov, G. Urbanik, K. Kummer, D. V. Vyalikh, O. V. Molodtsova, A. B. Preobrajenski, A. A. Zakharov, C. Hess, T. Hänke, B. Buchner, I. Vobornik, J. Fujii, G. Panaccione, Y. A. Ossipyan, M. Knupfer, Nano Letters 10 (3) (2010) 992. doi:10.1021/n1904115h.

[6] M. Cinchetti, K. Heimer, J.-P. Wüstenberg, O. Andreyev, M. Bauer, S. Lach, C. Ziegler, Y. Gao, M. Aeschlimann, Nature Materials 8 (2009) 115. doi:10.1038/nmat2334.

[7] V. Aristov, O. Molodtsova, Y. Ossipyan, B. Doyle, S. Nannarone, M. Knupfer, Organic Electronics 10 (2009) 8. doi:10.1016/j.orgel. 2008.09 .001$.

[8] J. C. Scott, Science 304 (2004) 62. doi:10.1126/science.1095520.

${ }_{220}^{2}$ [9] V. Y. Aristov, O. V. Molodtsova, C. Laubschat, V. M. Zhilin, I. M. Aristova, V. V. Kveder, M. Knupfer, Applied Physics Letters 97 (2010) -. doi:10.1063/1.3488809.

[10] R. Balog, M. Andersen, B. Jørgensen, Z. Sljivancanin, B. Hammer, A. Baraldi, R. Larciprete, P. Hofmann, L. Hornekær, S. Lizzit, ACS Nano 225 7 (5) (2013) 3823. doi:10.1021/nn400780x.

[11] A. Baraldi, G. Comelli, S. Lizzit, M. Kiskinova, G. Paolucci, Surface Science Reports 49 (2003) 169. doi:10.1016/S0167-5729(03)00013-X. 
[12] G. Ertl, Angewandte Chemie International Edition 47 (2008) 3524. doi: 10.1002/anie. 200800480.

[13] C. Kunze, D. Music, M. to Baben, J. M. Schneider, G. Grundmeier, Applied Surface Science 290 (2014) 504 - 508. doi:10.1016/j.apsusc.2013.11. 091.

[14] S. McDonnell, B. Brennan, E. Bursa, R. M. Wallace, K. Winkler, P. Baumann, Journal of Vacuum Science Technology B 32. doi:10.1116/1. 4878940.

[15] S. Yamamoto, I. Matsuda, Journal of the Physical Society of Japan 82 (2013) 021003. doi:10.7566/JPS J 82.021003.

[16] W. Widdra, D. Bröcker, T. Gießel, I. Hertel, W. Krüger, A. Liero, F. Noack, V. Petrov, D. Pop, P. Schmidt, R. Weber, I. Will, B. Winter, Surface Science 543 (2003) 87. doi:10.1016/j.susc.2003.07.005.

[17] P. Baumann, B. Krömker, G. Prümper, K. Winkler, R. Oiwa, J. Lenz, M. Maier, A. Feltz, conference proceedings, ALC 2011.

[18] A. Nambu, J.-M. Bussat, M. West, B. Sell, M. Watanabe, A. Kay, N. Mannella, B. Ludewigt, M. Press, B. Turko, G. Meddeler, G. Zizka, H. Spieler, H. van der Lippe, P. Denes, T. Ohta, Z. Hussain, C. Fadley, Journal of Electron Spectroscopy and Related Phenomena 137140 (2004) 691. doi:10.1016/j.elspec.2004.02.156.

[19] L. Kipp, Ph.D. thesis, Institut for Experimental and Applied Physics, University of Kiel (1992).

[20] E. Ludwig, H. Naggert, M. Kalläne, S. Rohlf, E. Kröger, A. Bannwarth, A. Quer, K. Rossnagel, L. Kipp, F. Tuczek, Angewandte Chemie (International Edition) 53 (2014) 3019. doi:10.1002/anie. 201307968.

[21] J. Viefhaus, F. Scholz, S. Deinert, L. Glaser, M. Ilchen, J. Seltmann, P. Walter, F. Siewert, Nuclear Instruments \& Methods in Physics Research A 710 (2013) 151. doi:10.1016/j.nima.2012.10.110. 
[22] F. Polack, M. Silly, C. Chauvet, B. Lagarde, N. Bergeard, M. Izquierdo, O. Chubar, D. Krizmancic, M. Ribbens, J.-P. Duval, C. Basset, S. Kubsky, F. Sirotti, AIP Conference Proceedings 1234 (2010) 185. doi:10.1063/1. 3463169.

[23] http://www.omicron.de/en/products/334/1054/.

[24] U. Vohrer, C. Blomfield, S. Page, A. Roberts, Applied Surface Science 252 (2005) 61. doi:10.1016/j.apsusc.2005.01.114.

[25] A. N. Chaika, O. V. Molodtsova, A. A. Zakharov, D. Marchenko, J. Sánchez-Barriga, A. Varykhalov, I. V. Shvets, V. Y. Aristov, Nano Research 6 (2013) 562. doi:10.1007/s12274-013-0331-9.

[26] A. N. Chaika, O. V. Molodtsova, A. A. Zakharov, D. Marchenko, J. Sánchez-Barriga, A. Varykhalov, S. V. Babenkov, M. Portail, M. Zielinski, B. E. Murphy, S. A. Krasnikov, O. Lübben, I. V. Shvets, V. Y. Aristov, Nanotechnology 25 (2014) 135605. doi:10.1088/0957-4484/25/13/ 135605.

[27] A. N. Chaika, N. N. Orlova, V. N. Semenov, E. Y. Postnova, S. A. Krasnikov, M. G. Lazarev, S. V. Chekmazov, V. Y. Aristov, V. G. Glebovsky, S. I. Bozhko, I. V. Shvets, Sci. Rep. 4 (2014) 3742. doi:10.1038/ srep03742.

[28] A. Grüneis, D. V. Vyalikh, Phys. Rev. B 77 (2008) 193401. doi:10.1103/ PhysRevB.77.193401.

[29] E. Rollings, G.-H. Gweon, S. Zhou, B. Mun, J. McChesney, B. Hussain, A. Fedorov, P. First, W. de Heer, A. Lanzara, Journal of Physics and Chemistry of Solids 67 (2006) 2172. doi:10.1016/j.jpcs.2006.05.010.

${ }_{280}^{20}$ [30] J. Hass, R. Feng, T. Li, X. Li, Z. Zong, W. A. de Heer, P. N. First, E. H. Conrad, C. A. Jeffrey, C. Berger, Applied Physics Letters 89 (2006) 143106. doi:10.1063/1.2358299. 
[31] C. Virojanadara, R. Yakimova, J. Osiecki, M. Syväjärvi, R. Uhrberg, L. Johansson, A. Zakharov, Surface Science 603 (2009) L87. doi:10.1016/j. susc. 2009.05 .005 .

[32] A. Ouerghi, M. Ridene, A. Balan, R. Belkhou, A. Barbier, N. Gogneau, M. Portail, A. Michon, S. Latil, P. Jegou, A. Shukla, Phys. Rev. B 83 (2011) 205429. doi:10.1103/PhysRevB.83.205429.

[33] K. V. Emtsev, A. Bostwick, K. Horn, J. Jobst, G. L. Kellogg, L. Ley, J. L. McChesney, T. Ohta, S. A. Reshanov, J. Röhrl, E. Rotenberg, A. K. Schmid, D. Waldmann, H. B. Weber, T. Seyller, Nature 8 (2009) 203. doi:10.1038/nmat2382. 